\title{
A novel active disturbance rejection controller for an electromagnetic valve actuator
}

\author{
Jiayu Lu and Siqin Chang \\ School of Mechanical Engineering, Nanjing University of Science and Technology, Nanjing, China
}

\begin{abstract}
In order to achieve fully variable and precise motion control of the electromagnetic valve actuator, enhance the engine performance, a novel active disturbance rejection controller for an electromagnetic valve actuator based on trajectory planning and acceleration feedforward is proposed. A fourth-order trajectory planning is used to achieve fully variable valve motion control, including variable valve lift and timing. It can also reduce the impact and vibration of EMVA system. Active disturbance rejection controller is used to estimate the variant dynamic and external disturbances of the system. The acceleration feedforward is compensated for the system to improve the tracking and steady state accuracy. Comparative simulations results show the proposed controller can improve the dynamic performance and the robustness of the system, and enhance the control precision.
\end{abstract}

\section{Introduction}

Camless engine is considered to be an efficient technology to improve the engine fuel economy, emissions and torque output performance. A novel electromagnetic valve actuator (EMVA) developed by the team of author's which has the characteristics of high response and large driving force is used on the camless engine. The valve lift, the valve duration, and the valve timing can be regulated flexibly by controlling the motions of the EMVA. And it can greatly improve the performance of the engine [1]. However the valve is directly driven by the EMVA, there is no intermediate link buffer, various disturbance factors are directly reflected on the system, which makes the dynamic characteristics of the system complex and changeable.

The main control goal of EMVA system is to achieve fully variable and precise motion control in spite of the various disturbance. Furthermore, soft landing control is required for the controller in order to guarantee working life of the gas regulating valve and reduce the working noise [2-5].

Active disturbance rejection controller (ADRC) is a new control method proposed by Han [6]. In ADRC, various known and unknown factors that influence the system performance are lumped together as total disturbance, and they are estimated and compensated in real time via an extended state observer (ESO), which is a key component of ADRC [7].

In this paper, a modified active disturbance rejection controller (MADRC) is proposed to meet the control requirements of EMVA system, including high control accuracy, robustness and anti-disturbance ability. A fourth-order trajectory planning is used to achieve fully variable valve motion control, including variable valve lift and timing. It can also reduce the impact and vibration of EMVA system. Active disturbance rejection controller is used to estimate the variant dynamic and external disturbances of the system. The acceleration feedforward is compensated for the system to improve the tracking and steady state accuracy. Simulation results indicate the effectiveness of the proposed controller.

\section{Structure and mathematical model of EMVA}

The structure of EMVA is shown in Fig. 1. The EMVA consists of a moving coil, permanent magnets, a valve, an inner core, and an outer core. The moving coil of EMVA is connected to the valve. The inner and outer core form a uniform air gap magnetic field. The movement of the valve can be controlled by controlling the magnitude and direction of the coil current [8].

The EMVA consists of electrical, magnetic, and mechanical subsystems, which can be described as follow

$$
\left\{\begin{array}{l}
\dot{x}=v \\
\dot{v}=\left(K_{m} / m\right) \cdot i-(K / m) \cdot x-F_{f} / m-F_{d} / m(1) \\
\dot{i}=-(R / L) \cdot i-\left(K_{e} / L\right) \cdot v+u / L
\end{array}\right.
$$

where $u$ is the input phase voltage. ${ }^{i}$ is the current through the coil. $R, L$ represent the resistance and inductance of the coil respectively. $m$ is the moving mass, $x$ denotes the mover position, $v$ is the velocity of the mover. $K_{m}$ is the force sensitivity if the actuator, which denotes the ratio of the electromagnetic force to the input current. $K_{e}$ is the back electromotive force coefficient. 
$F_{f}, F_{d}$ represent the friction and the external disturbance respectively, which is the uncertain nonlinearities of the system. Furthermore, in this EMVA, $K_{m}$ and $K_{e}$ are approximately equal in value.

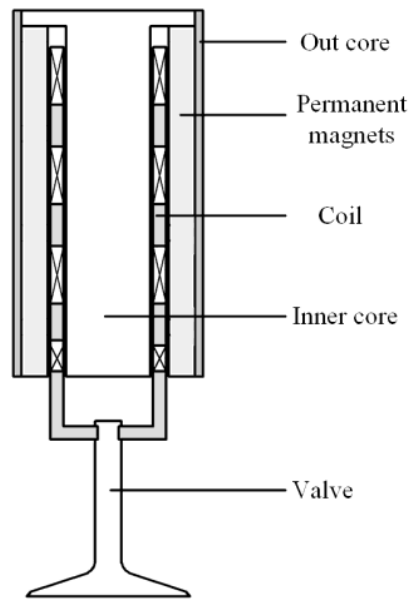

Figure 1. Structure of EMVA

\section{Control System Design of EMVA}

\subsection{Basic ADRC}

The basic ADRC consists of three parts: TrackingDifferentiator (TD), Extended State Observe (ESO) and Nonlinear Proportional-Derivative controller (NPD). Consider the following nonlinear second-order plant:

$$
\left\{\begin{array}{l}
\dot{x}_{1}=x_{2} \\
\dot{x}_{2}=f\left(x_{1}, x_{2}, d(t), t\right)+b \cdot u \\
y=x_{1}
\end{array}\right.
$$

where $y$ is the output to control, $u$ is the control input, $b$ is the system parameter, and $f\left(x_{1}, x_{2}, d(t), t\right)$ is the nonlinear total disturbance. The control objective is to make the output $y$ tracks the desired signal $y_{d}$ as far as possible in spite of the nonlinear disturbance.

The TD is used to arrange the transitional trajectory of the desired signal $y_{d}$.

ESO is the key component of ADRC, which can estimate the total disturbance. The discrete-time form of ESO for second-order plant Eq. (2) with sampling period $h$ is

$$
\left\{\begin{aligned}
e=z_{1}(k)-x(k) & \\
z_{1}(k+1)= & z_{1}(k)+h \cdot\left(z_{2}(k)-\beta_{01} \cdot e\right) \\
z_{2}(k+1)= & z_{2}(k)+h \cdot\left(z_{3}-\right. \\
& \left.\beta_{02} \cdot \operatorname{fal}\left(e, \alpha_{1}, \delta\right)+b \cdot i_{d}(k)\right) \\
z_{3}(k+1)= & z_{3}(k)-h \cdot \beta_{03} \cdot \operatorname{fal}\left(e, \alpha_{2}, \delta\right)
\end{aligned}\right.
$$

where $i_{d}$ is the control input of the second-order system, $\beta_{01}, \beta_{02}$ and $\beta_{03}$ are observer gains, $z_{1} 、 z_{2}$, and $z_{3}$ are estimates of the output $x$, the derivative of $x$, and the total disturbance respectively.

The nonlinear function $f a l(e, \alpha, \delta)$ is defined as

$$
f a l=\left\{\begin{array}{l}
e \cdot \delta^{\alpha-1}, \quad|e| \leq \delta \\
|e|^{\alpha} \cdot \operatorname{sgn}(e), \quad|e|>\delta
\end{array}\right.
$$

Parameter ${ }^{\alpha}$ satisfy condition $\alpha<1, \delta$ satisfy condition $\delta=j \cdot h$, where ${ }^{j}$ is a positive integer.

The indirect control input $u_{0}$ is determined by NPD which is given as

$$
\left\{\begin{array}{l}
e_{1}=y_{1}-z_{1} \\
e_{2}=y_{2}-z_{2} \\
u_{0}=\beta_{1} \operatorname{fal}\left(e_{1}, \alpha_{1}, \delta\right)+\beta_{2} \operatorname{fal}\left(e_{2}, \alpha_{2}, \delta\right)
\end{array}\right.
$$

where $\beta_{1}, \beta_{2}, \alpha_{1}, \alpha_{2}$ and $\delta$ are controller parameters.

Finally, the control input $u$ is given as

$$
u=\frac{u_{0}-z_{3}}{b} .
$$

\subsection{Proposed modified ADRC}

The transitional trajectory of the desired signal $y d$ arranged by TD is shown in Fig. 2 which is a kind of second-order profile planning.

As Fig. 2 shows, second-order profile planning can achieve good fastness of the control. However, the accelerated speed in the second-order profile planning is mutated which may cause the system to vibrate or oscillate, and it is not conducive to the precision control performance requirement. In order to solve this problem a forth-order profile planning is proposed. The forth-order profile planning can achieve smooth acceleration and deceleration, which can avoid mechanical shock and oscillate effectively. The typical forth-order profile planning for the point-to-point motion is shown in Fig. 3. $T_{0}$, s represent the movement time and lift of the EMVA respectively. dmax is the max jerk rate, td is the max jerk rate period. $t_{a}$ is the max acceleration period, and $t j$ is the max jerk period.

The use of sections to divide the text of the paper is optional and left as a decision for the author. Where the author wishes to divide the paper into sections the formatting shown in Table 2 should be used.

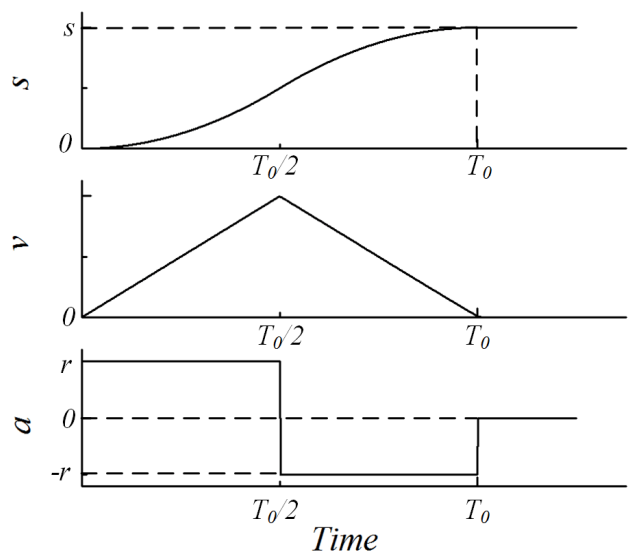

Figure 2. Transitional trajectory arranged by TD 


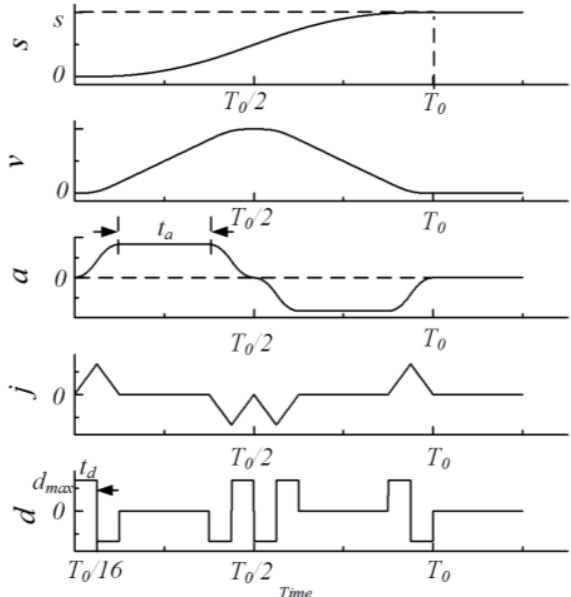

Figure 3. Typical forth-order profile planning

As Eq. (1) shows that the accelerated speed is mainly determined by the coil current, which is limited by the working voltage and actuator performance. Meanwhile, the max velocity of the actuator is not limited, according to [9], $t_{j}$ is tend to be zero. The movement distance $s$ can be given as

$$
s=\left(t_{d}{ }^{2} \cdot t_{a}{ }^{2}+6 t_{d}{ }^{3} \cdot t_{a}+8 t_{d}{ }^{4}\right) \cdot d_{\max } .
$$

In order to achieve the full flexibility control of the EMVA, $T_{0}, s$ should be variable. Let $t_{d}=T_{0} / 16$ and $t_{a}=T_{0} / 4$, so that $d_{\max }=4096 \cdot s /\left(3 \cdot T_{0}^{4}\right), d_{\max }$ is only determined by $T_{0}$ and $s$. The date of the trajectory planning is calculated by digital integration which can be described as

$$
\left\{\begin{array}{l}
j(t)=j_{0}+d_{\text {max }} \cdot t \\
a(t)=a_{0}+j_{0} \cdot t+d_{\text {max }} \cdot t^{2} / 2 \\
v(t)=v_{0}+a_{0} \cdot t+{ }_{0} j \cdot t^{2} / 2+d_{\text {max }} \cdot t^{3} / 6 \\
s(t)=s_{0}+v_{0} \cdot t+a_{0} \cdot t^{2} / 2+j_{0} \cdot t^{3} / 6+d_{\text {max }} \cdot t^{4} / 24
\end{array}\right.
$$

The feedback control is based on the system deviation which is caused by the output changes. So there exists timedelay in the feedback control. For the high speed and high precision control, the time delay of desired trajectory tracking may be more obvious and a steady-state error may be exist, if the simple feedback control is used. Meanwhile, the feedforward control can solve this problem. In order to improve the control performance of the system, a compound controller with feed-forward and feedback control is presented.

Fig. 4 shows the structure diagram of feedback controller, where $G_{c}(s), G_{p}(s)$ represent the controller transfer function and controlled plant transfer function respectively, $w$ is the total disturbance of the system, $z_{3}$ is the total disturbance which is estimated by the ESO. The system transfer function can be described as

$$
G_{1}(s)=\frac{G_{c}(s) \cdot G_{p}(s)}{1+G_{c}(s) \cdot G_{p}(s)}
$$

and the error transfer function of the system is

$$
E_{1}(s)=\frac{1}{1+G_{c}(s) \cdot G_{p}(s)}
$$

which is inevitable.

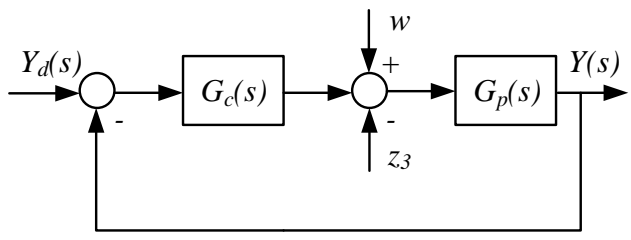

Figure 4. Structure diagram of feedback controller

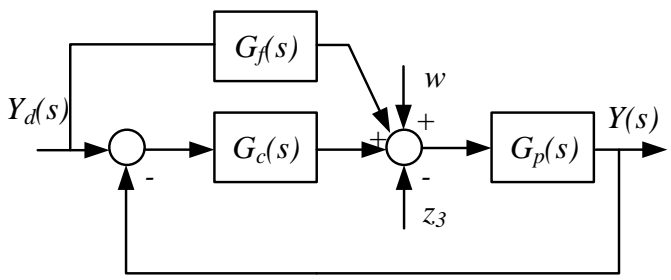

Figure 5. Structure diagram of compound controller

The structure diagram of compound controller with feedforward and feedback control is shown in Fig. 5. The system transfer function can be described as $G_{2}(s)=\frac{G_{c}(s) \cdot G_{p}(s)+G_{f}(s) \cdot G_{p}(s)}{1+G_{c}(s) \cdot G_{p}(s)}$, where $G_{f}(s)$ is the feedforward controller transfer function. And the error transfer function of the system is $\quad E_{2}(s)=\frac{1-G_{f}(s) \cdot G_{p}(s)}{1+G_{c}(s) \cdot G_{p}(s)}$ According to Eq. (6), if $z 3$ can completely estimate $w$, then $G_{p}(s)=\frac{1}{s^{2}}$. The feed-forward control transfer function is $s^{2}$, if acceleration feed-forward is used on the control system. So the error transfer function of the system tend to be zero, which proves that the compound controller can improve the control precision and does not affect the stability of the system.

The proposed control system of EMVA based on a novel ADRC consists of current and position loops, which is shown in Fig. 6. The modified ADRC is used in the position loop, the basic ADRC is implemented in the current loop. 


\section{Simulation results}

To test the effectiveness of the proposed modified ADRC, comparative simulations of the novel ADRC and traditional ADRC are implemented on EMVA with parameters given in Table I using MATLAB/Simulink.

\subsection{Comparative simulations}

he comparative simulations results are shown in Fig. 7. As Fig. 7(a) shows that both controllers have high steady precision, and there is no overshoot happened, but the MADRC achieves better performance for fast response. Fig. 7(b) is the tracking errors of the system, as we can observe in Fig. 7(b) that the max tracking error of ADRC and MADC is $159 \mu \mathrm{m}$ and $9 \mu \mathrm{m}$ respectively, which illustrates that the MADRC achieves better tracking performance. Fig. (c) is the velocity of EMVA.

It is demonstrated that a seating velocity (the average of three sampled valve velocities before the valve reaches the closed position) of $0.005 \mathrm{~m} / \mathrm{s}$ has been achieved in the MADRC system. And the average seating velocity for ADRC system is about $0.02 \mathrm{~m} / \mathrm{s}$, which illustrates that the MADRC achieves better soft landing performance. The control current of the system is shown in Fig. 7(d). As we can see that the control current of MADRC system is smoother than the ADRC system's, which may make the system more stable.

\subsection{Simulation results}

In order to achieve the fully variable control of the EMVA, the movement time T0, and lift s should be variable.

Fig. 8 (a) shows the system response with respect to various reference valve lift. The reference valve lifts are set to $4 \mathrm{~mm}, 6 \mathrm{~mm}$, and $8 \mathrm{~mm}$ respectively. The movement profile are planned by the fourth-order profile planning which is introduced in section 3.2.1. The movement time are set to $8 \mathrm{~ms}$. Fig. 8(b) shows the system response with respect to various movement time at the lift of $8 \mathrm{~mm}$. The movement times are set to $6 \mathrm{~ms}, 8$ $\mathrm{ms}$, and $10 \mathrm{~ms}$ respectively. Fig. 8 demonstrate that the MADRC achieves better performance for control precision and fast response, and the position error tends to be zero when MADRC is used in EMVA system.

Table 1. Parameters of the EMVA

\begin{tabular}{ll}
\hline Parameter & Valve \\
\hline$R$ & $1.38 \Omega$ \\
$L$ & $0.9 \mathrm{mH}$ \\
$K_{e}$ & $12 \mathrm{~V} \bullet \mathrm{s} / \mathrm{m}$ \\
$K_{m}$ & $12 \mathrm{~N} / \mathrm{A}$ \\
$m$ & $94.6 \mathrm{~g}$ \\
$u$ & $30 \mathrm{~V}$ \\
\hline
\end{tabular}

\subsection{Anti-disturbance ability analysis}

The EMVA suffers from various disturbances, such as combustion pressure and supercharge pressure. Since the disturbances are not well defined in the investigation, a step disturbance $\mathrm{F}$ is applied on the EMVA for a time 3-5 $\mathrm{ms}$ to estimate the disturbance rejection of the systems. Fig. 9 shows the simulation results when the values of $F$ is $\pm 100 \mathrm{~N}$. There is no overshoot happen in both ADRC and MADRC system. Fig. 9 (b)-(c) show the error of the system. Here error $=x-\bar{x}$, where $\mathrm{x}$ represents the trajectory obtained with control without disturbance, represents the trajectory obtained with control with disturbance. As Fig. 9 (b)-(c) shows that the MADRC has a smaller error than the basic ADRC, which shows that the MADRC has a better anti-disturbance ability than the basic ADRC.
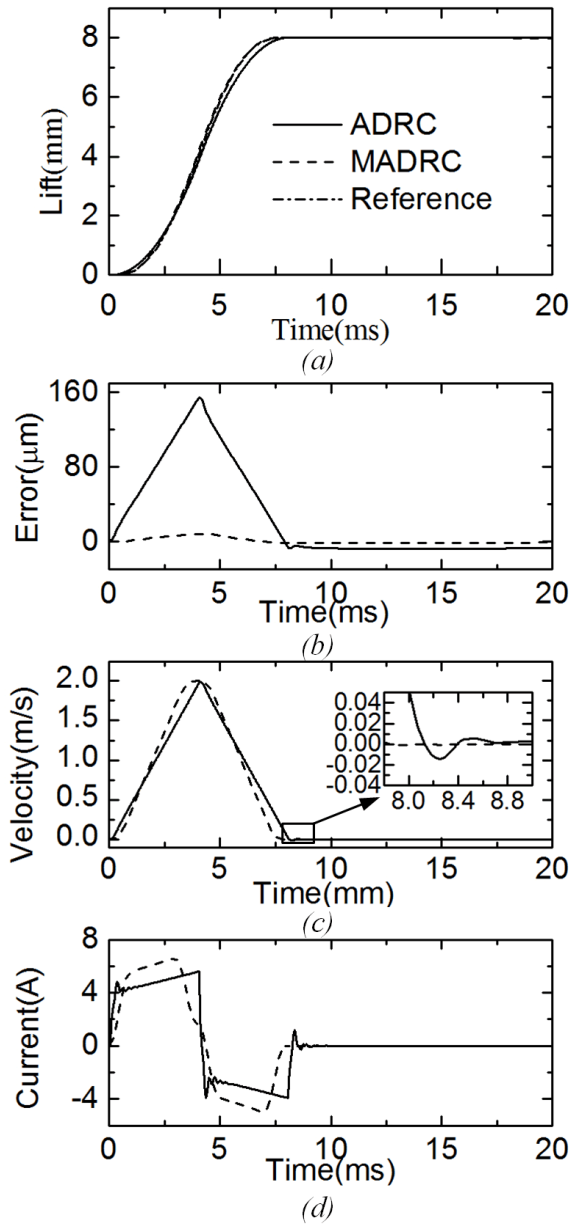

Figure 7. Comparative simulations results 

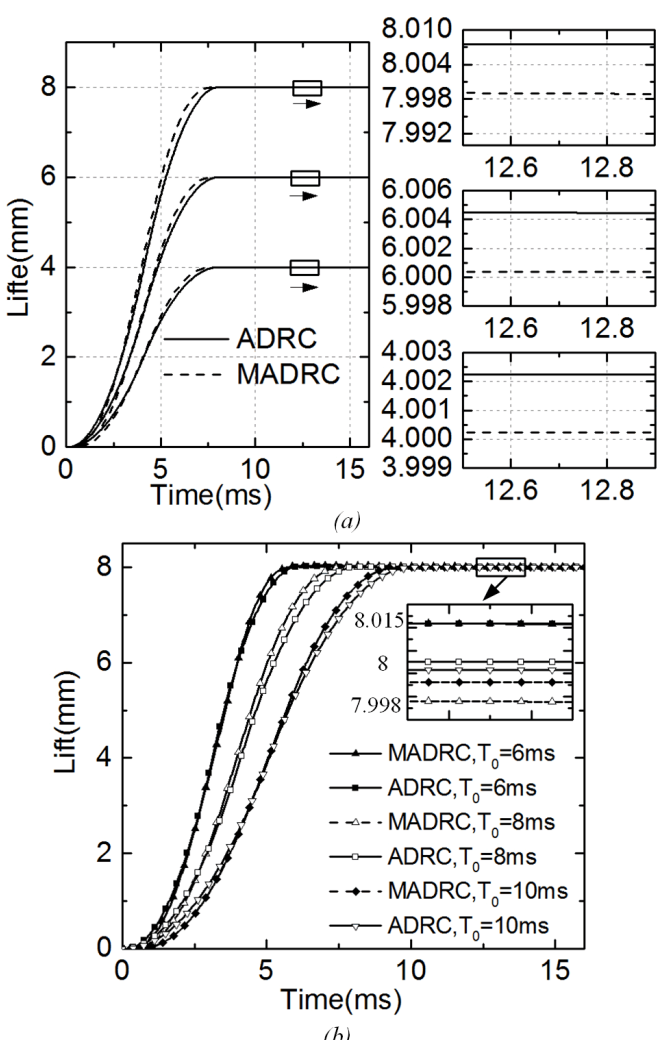

(b)

Figure 8. System response with various trajectory
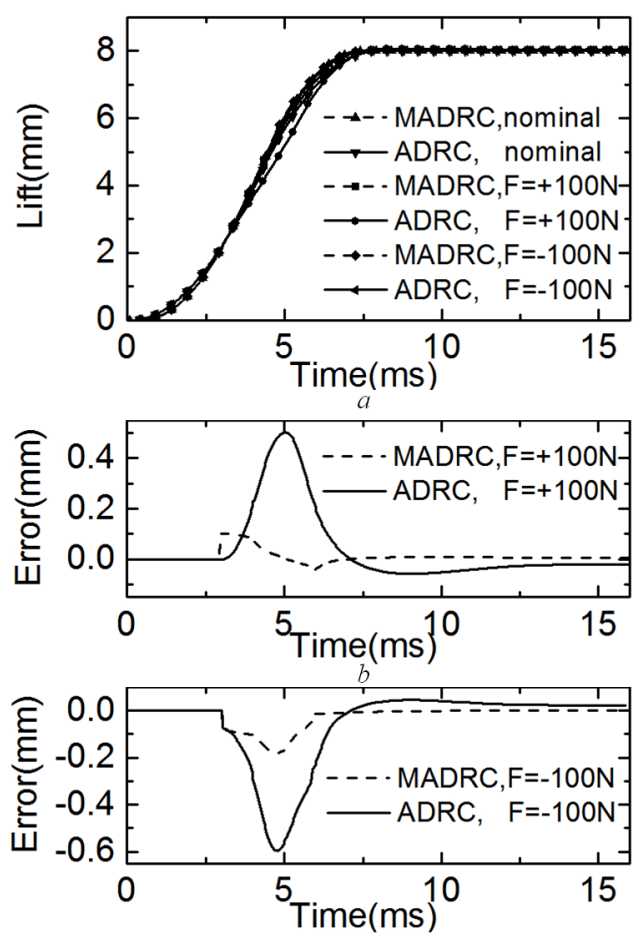

Figure 9. Disturbance rejection

\section{Conclusions}

A modified ADRC based on fourth-order trajectory planning and acceleration feedforward has been developed in this paper to achieve high-performance precision valve motion control. The proposed novel ADRC can achieve fully variable, including variable valve lift and timing, and soft landing, precise valve motion control and soft landing control. Meanwhile the proposed novel ADRC has strong robustness and antidisturbance ability. The performance of the proposed modified ADRC is verified by the comparative simulations. Consequently, if EMVA system with the proposed modified ADRC can be used on an automotive engine, it is predictably that the engine performance will be enhanced.

\section{References}

1. Chang, S., and L. Liu. A moving coil permanent magnet linear actuator with high power density, China patent, CN101127474B(2010)

2. Y Yang, Y. P., Liu, J. J., Ye, D. H., Chen, Y. R., \& Lu, P. H.. Multi-objective optimal design and soft landing control of an electromagnetic valve actuator for a camless engine. IEEE/ASME Transactions on Mechatronics, vol. 18, pp. 963-972(2013)

3. Mercorelli, P. Robust adaptive soft landing control of an electromagnetic valve actuator for camless engines. Asian Journal of Control, vol. 18, pp. 12991312 (2013)

4. Seethaler, R., Koch, C. R., Chladny, R., \& Mashkournia, M. Closed loop electromagnetic valve actuation motion control on a single cylinder engine. SAE Technical Papers, 2 (2013)

5. Paden, B. A., Snyder, S. T., Paden, B. E., \& Ricci, M. R. Modeling and control of an electromagnetic variable valve actuation system. IEEE/ASME Transactions on Mechatronics, 20(6), 26542665(2015)

6. Han, Jingqing, From PID to active disturbance rejection control, IEEE transactions on Industrial Electronics, vol. 56, pp. 900-906.

7. Shi, X., \& Chang, S.. Precision motion control of a novel electromagnetic linear actuator based on a modified active disturbance rejection controller. Proceedings of the Institution of Mechanical Engineers Part I Journal of Systems \& Control Engineering, vol. 226, pp. 606-614(2011)

8. Liu, L., \& Chang, S. Motion control of an electromagnetic valve actuator based on the inverse system method. Proceedings of the Institution of Mechanical Engineers Part D Journal of Automobile Engineering, vol. 226, pp. 85-93(2011)

9. Yang, Z. Research on fourth-order profile planning algorithm for high accuracy point-to-point motion system. China Mechanical Engineering, vol. 18, pp. 2346-2339(2007) 